\title{
Automated open wound suturing: detection and planning algorithm
}

\author{
Artur Sagitov ${ }^{1}$, Tatyana Tsoy ${ }^{1}$, Hongbing $\mathbf{L i}^{2}$, Evgeni Magid ${ }^{1}$ \\ ${ }^{1}$ Intelligent Robotics Department, Higher School of Information Technology and Information Systems, Kazan Federal \\ University, Kremlyovskaya str. 35, Kazan, Russian Federation \\ ${ }^{2}$ Department of Instrument Science and Engineering, Shanghai Jiao Tong University, Shanghai, China \\ E-mail:sagitov@it.kfu.ru,lihongbing@sjtu.edu.cn,tt@it.kfu.ru,dr.e.magid@ieee.org \\ http://kpfu.ru/robolab.html
}

\begin{abstract}
Today one of the key disadvantages of robotic surgery is a lack of a haptic feedback, while in traditional surgery a surgeon uses human haptic senses in all tasks. Providing robots with a haptic feedback have a high potential of reducing time that is spent by a surgeon on suturing subtasks and helping to reduce a surgeon fatigue. Decreasing manual input of a surgeon enables remote surgery even under long communication links with significant latency. In this paper we present a framework of wound detection and suture planning. We plan to implement and test our algorithms using KUKA iiwa manipulator.
\end{abstract}

Keywords: Robotics, robot assisted surgery, medical robotics, manipulator, algorithm, modelling, trajectory.

\section{Introduction}

A wound is defined as a body tissue injury that results into tissue continuity destruction ${ }^{1}$. Usually injuries are caused by physical means, and if a tissue had been significantly damaged beyond the level of noninterventional synulosis, an elaborated treatment procedure is required. The procedure includes cleaning up of foreign objects and dead tissue from a wound, infection treatment and wound suturing, that would allow further natural recovery of the wound. The tissue is maintained in apposition while natural healing processes strengthen the wound up to the level that it would withstand mechanical stress without additional support of sutures, strips, staples, or topical adhesives. An intervention that allows the wound to go through a repair process is referred as wound suturing ${ }^{2}$.

Robotic assistance may improve precision of surgical task performance as suturing is a challenging and timeconsuming task even for experienced surgeons. Zihni et al. ${ }^{3}$ showed that robotic assistance during intracorporeal suturing procedure decreased errors at all skill levels. Although it did not increase the task performance speed, some benefits in precision were noted.

Existing manual suture techniques could be transferred to automatic robot-assisted surgical suture, with all steps of suturing planned, approved and executed under surgeon expert supervision. Oquendo et $\mathrm{al}^{4}$ developed a hardware and software system to rate new laparoscopic suturing exercises automatically based on previously human assessed experiments. OSATS scores 5 of their system were comparable with a human expert assessment. The system facilitates more feedbackintensive surgical training and may yield insights into the fundamental components of surgical skills.

We target to implement procedures of automatic robot-assisted surgical suture using KUKA iiwa robot. The robot haptic feedback sensors would facilitate interactions with soft tissues. The algorithm plans a line of sutures to hold wound edges in apposition during a healing process. The line may consist of a single continuous strand or a series of interrupted suture strands. 
The paper concentrates on a procedure for automatic wound parameters detection and intervention planning, thus providing a first step toward the suture automation and extends our previous work ${ }^{6}$.

\section{Related Work}

Autonomous suturing with a help of surgical robots is a rapidly developing research field with challenges of transferring sensory-motor human skills to robotic manipulator systems. ${ }^{7}$ Previous studies examined various separate parts of a suture, including knot tying and thread tracking, or described an entire suturing procedure. Automated knot-tying for suturing with standard surgical instruments using skill transfer through a user demonstration was developed by Knoll et al. ${ }^{8}$ Every recorded user demonstration (a trajectory, sensory readings etc.) was decomposed into a chainable action primitives and a knot-tying task templates were reconstructed through feature extraction and fluid-based adaption of trajectories.

Apprenticeship learning approach with assumption that a trajectory should be smooth was demonstrated to autonomously execute learned trajectories for knot-tying with superior performance by Berg et al. for Berkeley Surgical Robots ${ }^{9}$. The experimental results showed that 10 times speedup was achieved comparing to average speed of the reference demonstrations.

Several autonomous knot tying algorithms with associated kinematic requirement were discussed by Kang and Wen implementing algorithms of collaborative operation of a surgeon and a robot ${ }^{10}$. Their suture control strategies included suture length modelling (tracking the effective length) and tension control, which required to secure a knot and was influenced by frictional forces.

Supervised automation of multi-throw suturing was presented by Sen et al., applying a custom low-cost mechanical needle guide to reduce needle pose uncertainty ${ }^{11}$. Using this guide and a real-time needle tracking system to provide a closed loop feedback, they were able to optimize process parameters (a trajectory and a needle type) to achieve multilateral needle hand-off for multi-throw suturing.

Osa et al. ${ }^{12}$ proposed an online trajectory planning and a force control system for surgery task automation that is applicable in real time suturing on a master-slave system with a velocity controller for combined spatial motion control and force tracking control. The task was modeled using Gaussian Process Regression with a conditional distribution of demonstrations, normalization in time domain and estimation with regard to a reference trajectory. Their experiments with tying a knot and cutting soft tissues showed that the system is operating in dynamic conditions robustly, including situations when a contact object changes its stiffness and position.

Jackson and Çavuşoğlu ${ }^{13}$ described development of an autonomous needle path planning for a simple interrupted suture based on well-established manual suture techniques. Suturing guidelines from surgeon manuals were analyzed yielding a parametrized path that specifies needle approach, bite, reorientation, regrasping and follow through. The path was optimized to minimize interaction forces between a tissue and the needle with proper enter/exit locations and orientations. Needle drive experiments estimated the forces and torques that were generated during a suture for both holonomic and nonholonomic drives.

Based on clinical suturing guidelines and using kinematic model of needle-tissue interaction with a surgeon that specified entry/exit points, Pedram et al. developed an algorithm for optimal selection of a needle diameter, shape, and constant curvature path ${ }^{14}$. The proposed algorithm was tested experimentally with the Raven II surgical system on a tissue phantom to guarantee minimal tissue trauma and compliance with suturing requirements.

Yet, all the above mentioned related work required surgeon input in selecting a suture path with a wound width and depth. Jackson and Cavusoglu presented a needle-tissue interaction model with friction force that was approximated as a static friction force ${ }^{15}$. The model was experimentally validated using a tissue phantom, allowing a proper modeling of a suture process.

Algorithms that use a stereo image for detection, initialization, and tracking of a suture thread through fitting a nonuniform rational B-spline (NURBS) curve allowed real time tracking a suture thread undergoing translations and deformation ${ }^{16}$. Chanda et al. ${ }^{17}$ presented a review for computational modeling of wound suture and closure mechanics with geometrical modeling, material characterizations, finite element meshing.

\section{Suturing procedures}

Our research focuses at open skin wounds. Skin consists of an epithelium and an underlying dermis, which require sharp stitching needle in order to minimize tissue trauma. Sutures are required to withstand a natural skin tension 
while holding wound edges in apposition. A skin closure suturing technique could be continuous or interrupted, but the latter is usually preferred ${ }^{18}$. Each suture strand is passes only once through the skin, thus reducing a chance of a cross-contamination across the entire suture line. The following factors affect successful suturing intervention:

Closing tension. Tension in the suture should be sufficient to approximate tissue and eliminate dead space, while being loose enough to prevent tissue ischemia, necrosis and patient discomfort. Therefore, a proper control for closing tension is required.

Needle. A surgical needle is attached to a suture strand to permit repeated passes through the tissue while suturing tissues. An algorithm should integrate particular needle type parameters into a preplanning procedure because multiple variety of needles may be applied.

Suture. A suture is a strand of material being used to pull tissue edges together. A suture is characterized with several parameters including suture material size, a knot tensile strength, and a number of strands. In practice the smallest diameter suture that could hold the mending wounded tissue is utilized in order to minimize tissue trauma. Knot tensile strength corresponds to a force that a suture strand can withstand before it breaks while being knotted. The suture tensile strength should not surpass the tissue tensile strength, but the sutures should be at least as strong as regular tissue of wound edges ${ }^{18}$. The material size is measured numerically, while both strand diameter and tensile strength decrease proportionally to the suture size decrease. Sutures are classified according to a number of strands they contain. A monofilament suture contains a single strand of material and encounter less resistance as it passes through a tissue comparing to a multifilament suture. Multifilament sutures consist of several strands, twisted or braided together to increase tensile strength, pliability, and flexibility.

Knot tying. Each knot that is placed for tissues approximation should be tied with a high precision and held with a proper tension. A particular knot type to be used depends on the suture, the depth, and the amount of stress that will be placed postoperatively. After the knot is tied, the ends of a surgical gut should be cut.

From wound geometry, wound depth and suture material properties we can estimate and characterize the suture force requirements accurately. For most areas of the body, except a human face, sutures should be placed into skin $3-4 \mathrm{~mm}$ from a wound edge and $5-10 \mathrm{~mm}$ apart $^{19}$.

\subsection{Suturing techniques}

There are several types of suture techniques. With regard to continuity we distinguish continuous and interrupted sutures. Continuous sutures are made with a single strand that is connected to itself at each end, or with both ends being connected together. As mechanical strength comes from tension being distributed over all strand length, we should produce enough tension, while avoiding excess tension that leads to tissue strangulation. Excessive tension can also lead to full suture failure. Interrupted sutures combine multiple strands to close a wound with each stich being tied separately after a procedure completion. This provides a more secure closure, as while individual stitch breaks, the rest of the stitches would hold the wound edges close to each other. Our long term goal considers continuous and interrupted sutures with a dynamic model of a surgical thread.
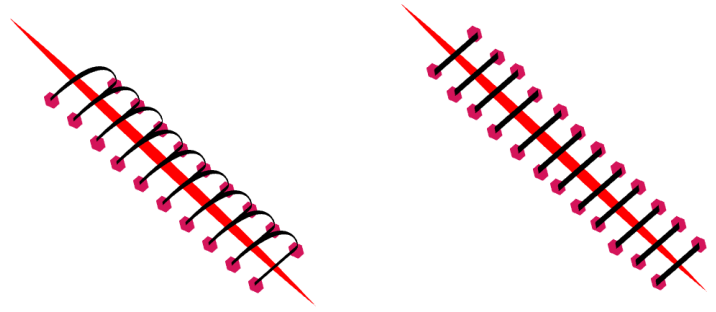

Fig. 1. Continuous (left) and interrupted (right) suture models.

Other types of primary sutures, such as deep sutures, buried sutures, purse-string sutures, and subcuticular sutures, are used for specific purposes. Deep sutures may be continuous or interrupted sutures, which are entirely hidden under an epidermal skin layer, and are not further removed postoperatively. Buried sutures are placed with a knot protruding to the inside, under a layer, which is to be closed. Purse-string sutures are continuous sutures being placed around a lumen and tightened like a drawstring to invert the opening.

\subsection{Suturing algorithm overview}

Figure 2 describes a suturing procedure algorithm. An image from a robot camera is captured, dimensional parameters of a wound are estimated and a supervising surgeon specifies suture technique parameters (interval and spacing) and material to be applied. The robot generates an intervention initial plan that sets up an exit/entry and a knot point locations. Based upon calculated points and formalized suture techniques an individual point to point needle path planning is 
performed. The suture plan is delivered to the surgeon for a confirmation, and the plan is executed under his/her supervision. Sensory data and trajectories are recorded for post operational analysis for further use in training, best practices selection, errors and malfunctions recording, quality control, and possible independent audit of the entire operation. Finally, the suture undergoes quality control by the supervising surgeon.

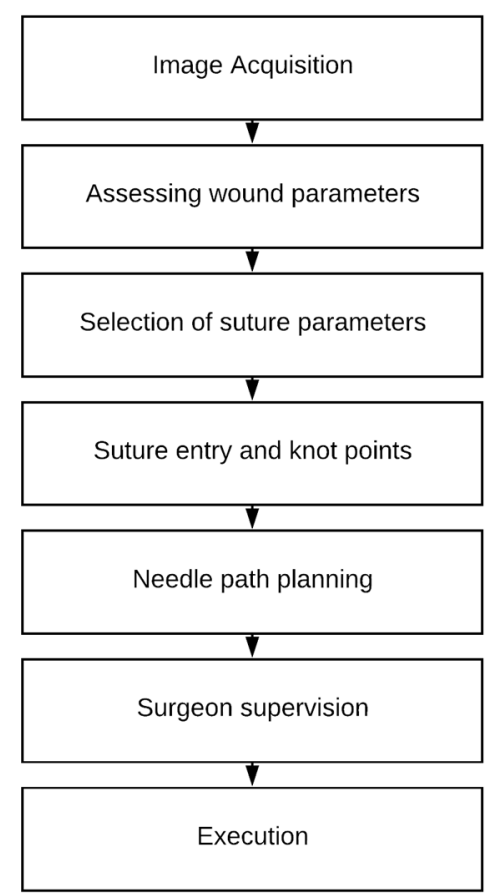

Fig. 2. Automatic suture procedure.

\subsection{Wound detection and exit/entry points selection}

Using an image from an operation area camera, suturing procedure parameters are estimated. Since a wound is a skin irregularity, a clustering procedure allows possible wound locations detection. The algorithm for wound boundaries and locations of exit/entry points estimating works as follows (Fig.3):

1. Low-pass filtering averages out rapid changes in intensity;

2. Mean shift clustering method is used to extract cluster components;

3. Clusters that are wound representations are selected from cluster components;

4. Feature points are extracted from a wound cluster image using a topological skeleton transform (medial axis transform);
5. A wound centerline approximation spline is constructed;

6. An upper and a lower wound suture splines are constructed by placing points tangentially to local vicinity by stich width;

7. Exit/entry points are generated with respect to suture model and placed on boundary splines;

8. Point processing order is assigned according to a selected suturing technique;

9. Knotting points are added at the ends of each continuous stitch.

The combination of ordered list of entry/exit points and knots comprise our initial suture plan.
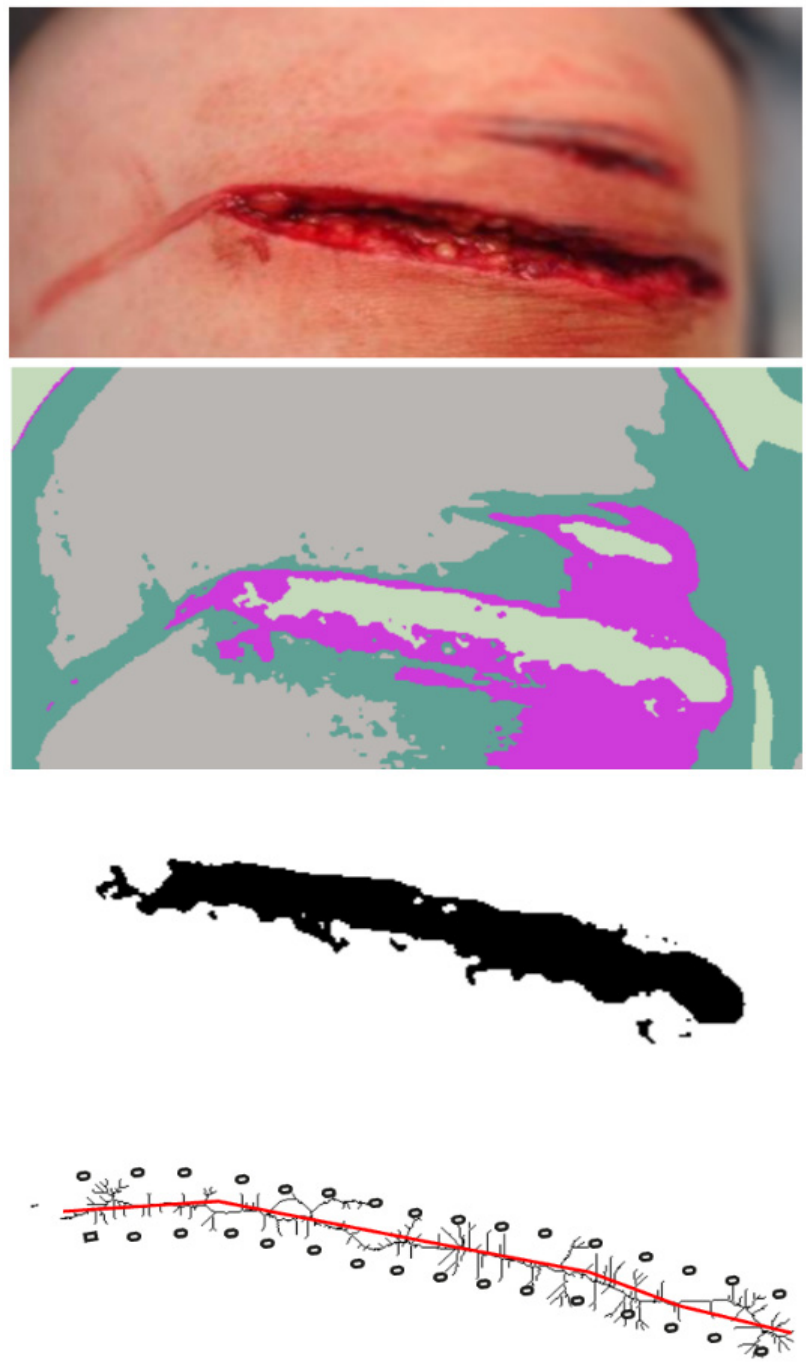

Fig. 3. Wound borders and exit/entry point evaluated step by step for a continuous suture. The input photo (top) is obtained from www.thinglink.com/scene/911241389793083393. 


\subsection{Process supervision}

The automatically generated initial suture plan is presented to a supervising surgeon, which can modify the plan by moving/adding/deleting points/knots and changing the order of stitching. After the surgeon approval the assisting robot can proceed to plan and execute individual point operations, while the surgeon continuously observes a current action of the robot, next actions' plan and suture tension. Moreover, he/she is authorized to correct the robot at any moment. All actions of the assisting robot and sensory data are carefully recorded for future analysis. Using artificial machine intelligence, we can improve outcomes of surgical procedures and significantly reduce surgeon fatigue.

\section{Conclusions}

This paper presented initial stages of autonomous supervised suturing including a wound boundary detection and exit/entry points generation, that are followed by knot points allocation, being specific to particular selected technique and parameters. Our future work is to adopt the existing methods in needle path planning and integrate them into our framework to evaluate performance on KUKA iiwa robot using haptic feedback and a ZED camera stereo vision for a wound profile reconstruction.

\section{Acknowledgements}

This work was supported by the Russian Foundation for Basic Research (RFBR) and the Government of the Republic of Tatarstan, project ID 18-48-160037. Part of the work was performed according to the Russian Government Program of Competitive Growth of Kazan Federal University.

\section{References}

1. M. Richardson, Acute wounds: an overview of the physiological healing process, Nursing times, 100 (4), 2003, pp. 50-53.

2. G. C. Gurtner, S. Werner, Y. Barrandon, and M. T. Longaker, Wound repair and $\mathrm{r}$ egeneration, Nature, $\mathbf{4 5 3}$ (7193), 2008, pp. 314-321.

3. A. Zihni et al., Comparison of precision and speed in laparoscopic and robot-assisted surgical task performance, J. of Surgical Research, 223, 2018, pp. 29-33.

4. Y. A. Oquendo, E. W. Riddle, D. Hiller, T. A. Blinman and K. J. Kuchenbecker, Automatically rating trainee skill at a pediatric laparoscopic suturing task. Surgical Endoscopy 32 (4), 2018, pp. 1840-1857.
5. J. A. Martin, G. Regehr, R. Reznick, H. Macrae, , J. Murnaghan, C. Hutchison and M. Brown, Objective structured assessment of technical skill (OSATS) for surgical residents. British J. of surgery, 84 (2), 1997, pp. 273-278.

6. A. Sagitov, H. Li and E. Magid, Toward automated open wound suturing using haptic feedback: detecting wounds and planning the suture. Int. Conf. on Artificial Life and Robotics, 2018, pp. 208-211.

7. M. Yip and N. Das, Robot autonomy for surgery. arXiv preprint arXiv:1707.03080, 2017.

8. A. Knoll, H. Mayer, C. Staub, and R. Bauernschmitt, Selective automation and skill transfer in medical robotics: a demonstration on surgical knot-tying, The Int. J. of Medical Robotics and Computer Assisted Surgery 8 (4), 2012, pp. 384-397.

9. J. Van Den Berg, S. Miller, D. Duckworth, H. Hu, A. Wan, X.-Y. Fu, K. Goldberg, and P. Abbeel, Superhuman performance of surgical tasks by robots using iterative learning from human-guided demonstrations, Proc. of IEEE Int. Conf. on Robotics and Automation, 2010, pp. 2074-2081.

10. H. Kang and J. T. Wen, Autonomous suturing using minimally invasive surgical robots, Int. Conf. on Control Applications (,2000), pp. 742-747.

11. S. Sen, A. Garg, D. V. Gealy, S. McKinley, Y. Jen and K. Goldberg, Automating multi-throw multilateral surgical suturing with a mechanical needle guide and sequential convex optimization, IEEE Int. Conf. on Robotics and Automation, 2016, pp. 4178-4185.

12. T. Osa, N. Sugita and M. Mitsuishi, Online Trajectory Planning and Force Control for Automation of Surgical Tasks, IEEE Trans. on Automation Science and Engineering 15 (2), 2018, pp. 675-691.

13. R. C. Jackson and M. C. Çavuşoğlu, Needle path planning for autonomous robotic surgical suturing, IEEE Int. Conf. on Robotics and Automation, 2013, pp. 1669-1675.

14. S. A. Pedram, P. Ferguson, J. Ma, E. Dutson and J. Rosen, Autonomous suturing via surgical robot: An algorithm for optimal selection of needle diameter, shape, and path, IEEE Int. Conf. on Robotics and Automation, 2017), pp. 2391-2398.

15. R. C. Jackson and M. C. Çavuşoğlu, Modeling of needletissue interaction forces during surgical suturing, IEEE Int. Conf. on Robotics and Automation, 2012, pp. 4675-4680.

16. R. C. Jackson, R. Yuan, D. Chow, W. S. Newman and M. C. Çavuşoğlu, Real-Time Visual Tracking of Dynamic Surgical Suture Threads, IEEE Trans. on Automation Science and Engineering, 15 (3), 2018, pp. 1078-1090.

17. A. Chanda, T. Ruchti and V. Unnikrishnan, Computational Modeling of Wound Suture: A Review, IEEE Reviews in Biomedical Engineering, 11, 2018, pp. 165-176.

18. D. L. Dunn and J. Phillips, Wound closure manual, Ethicon, Somerville, New Jersey, 2007.

19. N. B. Semer. Practical plastic surgery for nonsurgeons, iUniverse, 2007. 